\title{
Optimization of video quality via fuzzy logic base adaptive media playout
}

\author{
Farij Ehtiba' $^{1}$ Badlishah Ahmad ${ }^{2}$, Mostafijur Rahman ${ }^{3}$ \\ ${ }^{1}$ Department of Communication and Computer Networks, Inforrmation Technology School, Misurata University, Libay \\ ${ }^{2}$ ENAC Research, School of Computer and Communication Engineering, University Malaysia Perlis, Malaysia \\ ${ }^{3}$ Department of Software Engineering, Daffodil International University (DIU), Dhaka, Bangladesh
}

\begin{tabular}{l} 
Article Info \\
\hline Article history: \\
Received Sep 9, 2018 \\
Revised Nov 10, 2018 \\
Accepted Nov 24, 2018 \\
\hline Keywords: \\
Adaptive media playout \\
Fuzzy logic \\
Video streaming \\
Virable bit rate
\end{tabular}

\begin{abstract}
This paper addresses the issue of interruption of Virable Bit Rate (VBR) video streaming over IP network, due to channel quality fluctuation. Specifically, a Fuzzy Logic (FL) Control principle is fused with Adaptive Media Playout (AMP) control to estimate an appropriate playout frame rate, namely FLAMP. Based on the estimation of the playout frame rate, the FLAMP algorithm adjusts the consumption rate smoothly to avoid video stream degradation. Simulation results validate that the FLAMP scheme efficiently reduces the buffer outage probability and provides better visual quality where FLAMP gives $26.5 \%$ less ferquence of playout interruption, and $21.6 \%$ less variance of distortion of playout as compared to APTA.
\end{abstract}

Copyright () 2019 Institute of Advanced Engineering and Science. All rights reserved.

\section{Corresponding Author:}

Farij Ehtiba,

Department of Communication and Computer Networks,

Inforrmation Technology School,

Misurata University, Libay.

Email: F.ehtiba@it.misurratau.edu.ly

\section{INTRODUCTION}

Video conference, video on Demand (CoD) and Internet Protocol Television (IPTV) are good examples of multimedia that reflects a boom of media streaming services over the Internet [1]. In general, video streaming is transmitted to the client in a continuous manner, which can be watched immedatly as it received. However, the delivery of video streaming over IP networks still faces many challenges due to variable bandwidth which casues delay jitter, and packet loss [2,3].

Commonly, data buffering is placed at the client to alleviate the degradation of network quality. Practically, some video frames are stored in the received buffer to relieve the playout interruptions resulting from variable bandwidth. Thus, the receiver buffer is monitored for triggering the adaptive of playout rate. Aggressively adoption technique, which consists of simply discarding or duplicated video frame, is used to adjust the playout frame rate. Even though the probability of playout degradation decreases as more video frames are buffered, the playout delay increases [4-6]. On the other hand, Aggressively adoption can cause playout disruptions or discontinuities with a consequent degradation of the Quality of Experience (QoE) [7].

AMP reduces playout delay by minimize the storage data and adjusts playout rrate based on channel quality [8]. AMP techniques are smooth adjustment techniques based on changing the playout rate within acceptable ranges. In general, the objective of the existing AMP techniques is to adapt the playout rate to compensate the deviation between the arrival rate and consumption rate. However, most of the existing AMP techniques are implemented by two main steps, triggering playout rate and interpolation playout rate. Regarding to the interpolation playout rate, lots AMP techniques are based on linear playout adjustment [9- 
12]. In contrast, a quadratic playout adjustment is porposed in [13] which gives better performance in term of reducing the distortion of the playout curve.

Since the playout curve smoothness is a main key of satisfying the QoE, a new technique, namely FLAMP, is proposed in this paper to investigate and demonstrate that AMP based on Fuzzy logic control gives a good performance in terms of minimizing the probability of buffer underflow, the ratio of buffer overflow, the variation of playout of distortion and abrupt variations in the playout curve.

\section{DESIGN OF FLAMP TECHNIQUE}

\subsection{System Model}

Figure 1 illustrates the architecture of typical video streaming design over IP networks. The video stream originating from a streaming server reaches the client, and queued to be displayed.

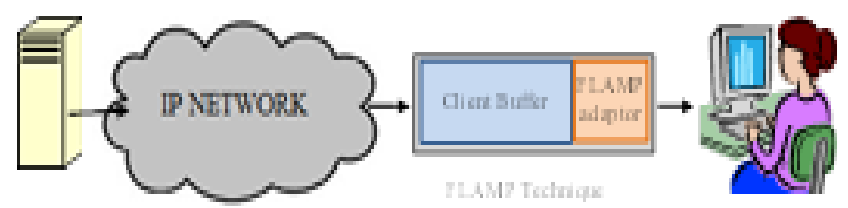

Figure 1. Typical architecture of FLAMP technique video streaming over IP network

The client has the ability to manipulate the frame duration $\mathrm{d} \in \mathrm{S}=\{\mathrm{d} 1, \mathrm{~d} 2, \ldots, \mathrm{dn}\}$, where $\mathrm{d} 1>\mathrm{d} 2,>\cdots>$ $\mathrm{dn}$ and $\mathrm{dn}$ is the normal video frame separation. However, The time of playout is divided into time slots which have same time durations of $\mathrm{t}=\mathrm{dn}$. When the video frames reaches the client's buffer are queued for displaying later. Let $A n \in A=\{0,1, \ldots, A n\}$ denotes the number of arrival frame during time slot $t$, where An is the maximum number of arrival frames.

Since the channel quality is fluctuated and the inter arrivals of video frames are variable, the frame arrival process may be further randomize. Let $D_{n} \in D=\left\{1,2, \ldots, D_{n}\right\}$ denote the number of frames displayed during slot $t$. Define furthermore $\mathrm{B}_{L V L}$ as the buffer fullness level in terms of the number of frames at the beginning of slot $\mathrm{t}$. Then the next buffer fullness level $\mathrm{B}_{L V L+1}$ and its deviation is categorized as:

$$
\begin{aligned}
& B_{L V L}=B_{L V L-1}+\left(A_{n}-D_{n}\right) \\
& B_{V A R}=B_{L V L}-B_{L V L-1}
\end{aligned}
$$

The frame playout duration (d) is constrained, which represents the time interval to be increased or decreased in a single step according to channel quality. Subsequently, the concept of FL control is fused with AMP to tune the interval time $\left(\mathrm{P}_{\text {OUT }}\right)$ and adapts playout rate accurately. The goal of FLAMP design is to manipulate the frame playout to compensate the deviation between consumption rate and arrival rate.

\subsection{System Model}

The architecture of FLAMP control technique is presented in Figure 2. It contains of two inputs, buffer fullness $\left(\mathrm{B}_{L V L}\right)$ and variance of buffer fullness $\left(\mathrm{B}_{V A R}\right)$, are chosen as inputs for the FL control to tune the playout rate. Firstly, the input variables are converted into suitable linguistic variables. Secondly, the linguistic variables are emulated based on the knowledge base to make accurate decisions. Finally, the result of Fuzzy Inference System (FIS) is defuzzified into a crisp value which represents the accurate interval time $\left(\mathrm{P}_{\text {OUT }}\right)$ to display the next video frame. 

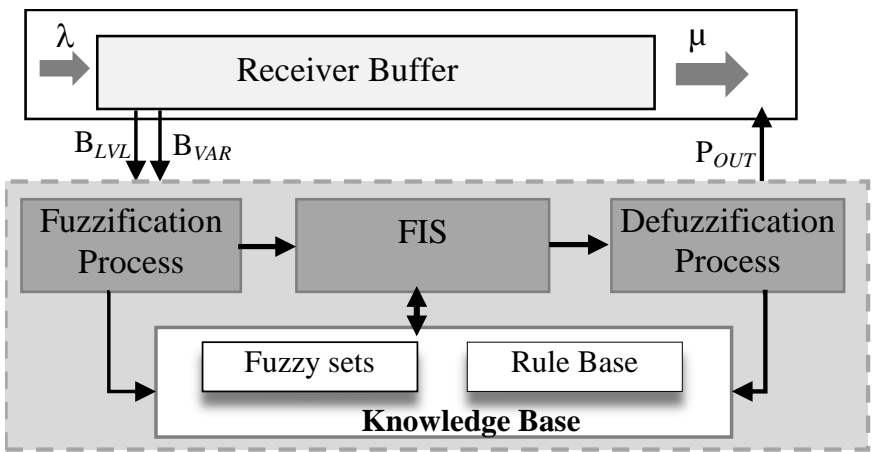

Figure 2. Effects of selecting different switching under dynamic condition

\subsubsection{Fuzzy Sets Membership of FLAMP Technique}

A fuzzy set is defined as a group of elements with a continuum of membership grades. Each element has a grade of membership ranging between [0-1]. In FLAMP technique, two input parameters, Figures 3 and 4 , and an output parameter, Figures 5, are utilized to regulate the playout rate. However, the input and output parameters are used to define the five linguistic variables as shown in Table 1.

Table 1. Inputs and Output Linguistic Variables of FLAMP Fuzzy Sets Membership

\begin{tabular}{cc}
\hline Variable & Fuzzy Set Membership \\
\hline$B_{\text {LVL }}$ & [V-Low, Low, Normal, High, V-High] \\
$B_{V A R}$ & [N-High, N-Low, Normal, P-Low, P-High] \\
$P_{\text {OUT }}$ & [V-Low, Low, Normal, High, V-High] \\
\hline
\end{tabular}

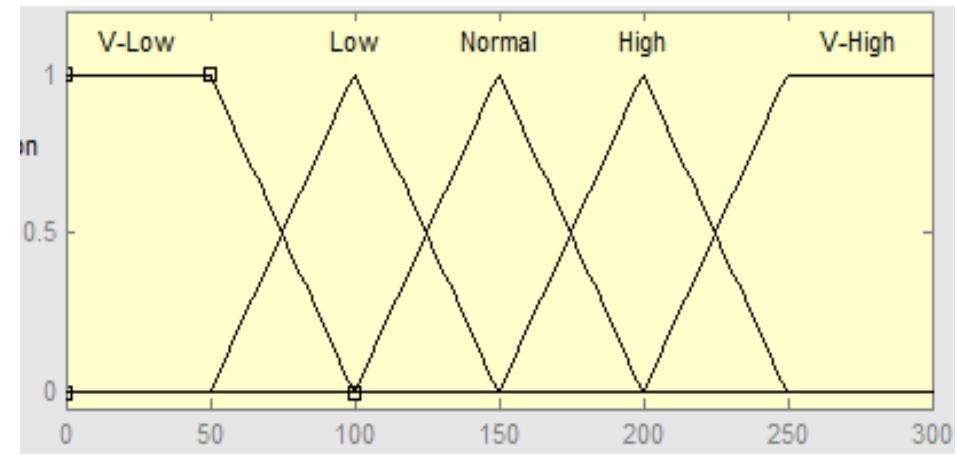

Figure 3. The membership function of buffer fullness level $\left(\mathrm{B}_{L V L}\right)$

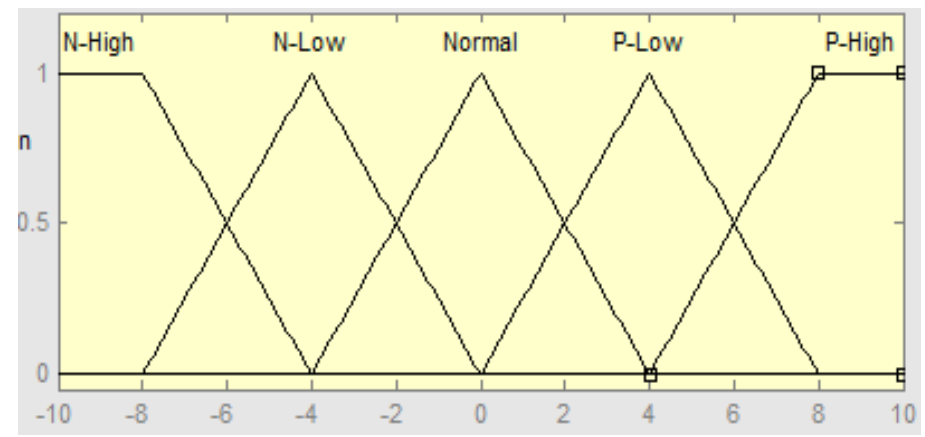

Figure 4. The membership function of buffer fullness level ( $\left.\mathrm{B}_{V A R}\right)$ 


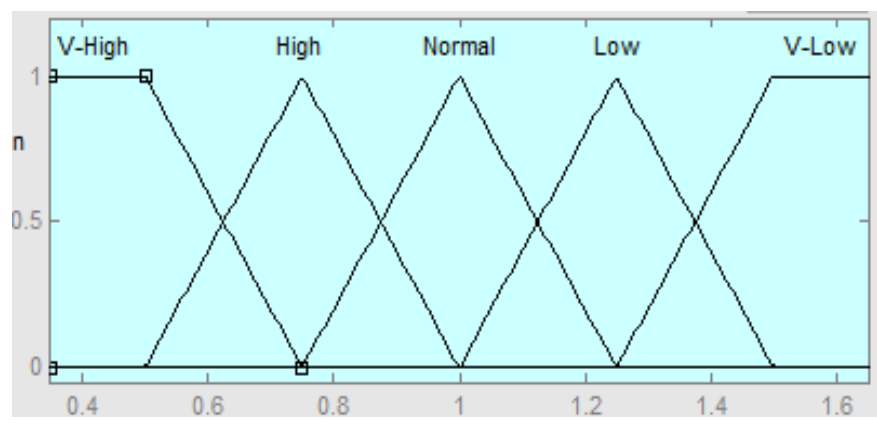

Figure 5. The membership function of playout rate $\left(\mathrm{P}_{\text {OUT }}\right)$

\subsubsection{The FLAMP Technique Rule-Base}

The FLAMP rule-base consists of group of fuzzy rules. They use to formulate the conditional statements that comprise fuzzy logic. The philosophy of the FLAMP technique rule is to adapt the playout rate when the deviation between arrival rate and consuming rate occurs. Table 2 illustrates the IF-Then rules of the FLAMP technique which the column represents the fuzzy set of buffer fullness and the row represents the fuzzy set of the variation of buffer fullness.

Table 2. The Rule-Base of FLAMP Technique

\begin{tabular}{ccccccc}
\hline \multirow{2}{*}{ Variables } & \multicolumn{5}{c}{ Buffer Variation $\left(\mathrm{B}_{V A R}\right)$} \\
& & Negative High & Negative Low & Normal & Positive Low & Positive High \\
\hline & Very Low & Very Low & Very Low & Very Low & Very Low & Very Low \\
Buffer Fullness & Low & Very Low & Low & Low & Low & Normal \\
Level $\left(\mathrm{B}_{L V L}\right)$ & Normal & Low & Normal & Normal & Normal & High \\
& High & Normal & High & High & High & Very High \\
& Very High & Very High & Very High & Very High & Very High & Very High \\
\hline
\end{tabular}

\subsubsection{Fuzzy Inference Process (FIP) of FLAMP}

FIP, the kernel of a fuzzy logic system, is the process of formulating the mapping from the given inputs to an output using fuzzy logic. Since Mamdani-based model is a commonly used as fuzzy methodology, it has been chosen as the methodology of the FLAMP controller [14]. The FLAMP technique involves four stages [15]. The FIS employs buffer fullness and its variation to tune the perfect playout rate as follows.

The first step is to determine the membership degree of the input variables $\left(\mathrm{B}_{L V L}\right.$ and $\left.\mathrm{B}_{V A R}\right)$, where the deviation of buffer fullness is calculated as shown (2): The second step is to evaluate the FLAMP rules of the. The degree of membership of each preceding input variable is assessed using the rules-base of the FLAMP technique. The third step is to aggregate the rulers' outputs. The membership values of all rules are compound into an output value.

The fourth step is the Defuzification. The aggregate output fuzzy set is defuzzified to obtain a single number which represents the new playout interval $\left(\mathrm{P}_{\text {OUT }}\right)$. The center of gravity $(\mathrm{COG})$ method is chosen defuzification process. Formally, the COG is calculated as follows.

$$
C O G=\frac{\sum_{a}^{b} F_{S}(S) \times S}{\sum_{a}^{b} F_{S}(S)}
$$

\section{EXPERIMENTS AND RESULTS}

This section characterizes and compares the performance of the FLAMP technique with previous techniques. The simulation settings and the video stream are described. To evaluate the performance of control techniques, the metrics are designated. Finally, the simulation results of all technique are discussed and compared. For the FLAMP technique validation, various AMP techniques have been examined. 
a) No Control: the video stream is displayed back without adaptition control.

b) Linear Slowdown and Speedup: The playout adaptation control, which speedup and slow down playout rate, is performed to adjust playout rate according to threshold level [8].

c) APTA technique: The APTA algorithm is employed to validate the proposed techniques. The APTA algorithm is a threshold base technique which the playout rate is quadratically decreased when the data level is below low threshold. In contrast, it is quadratically increased when the data level exceeds high threshold. Otherwise, it is adapted depending on the instant arrival rate [13]. Furthermore, the APTA parameters are set as in [13].

\subsection{Simulation Setting}

Network Simulator 2 (NS2) is adopted to validate the FLAMP technique [14]. Accordingly, the MPEG-4 encoded video, Jurassic Park I [15] is obtained as a video stream source. The video packets which are sent over IP network to the client is jittered with different patterns. Since Pareto ON-OFF source can jitter video stream more significantly than others, a Pareto with parameters (400 ms, $600 \mathrm{~ms})$ has been chosen to jitter the video stream [9]. The arrival time of all packets are stored and used as the arrival process of the AMP techniques.

\subsection{Performance Metrics}

The variation of playback speed affects the video streaming playout, which causes degradation of video quality. Therefore, several effective metrics for AMP techniques are defined as the following.

\subsubsection{Frequency of Playout Interruptions}

The video stream is interrupted when there is no frame in the client's buffer. Naturally, less frequency of playout interruptions is better performance.

$$
f_{U}=\frac{N_{U}}{T}
$$

where $N_{U}$ represents the number of playout interruptions during play the video clip time T.

\subsubsection{Variance of Distortion of Playout (VDoP)}

Playout interruption strongly affects the quality of experience (QoE). The distortion of playout (DoP) is presented in [13] to study the effects of playout interruption on video streaming.

$$
V D o P=\frac{\sum_{i=1}^{N} D o P^{2}(i)}{N}-\overline{D o P}^{2}
$$

where $\mathrm{N}$ represents total received frames including discarded frames.

\subsubsection{Playout Curve}

Playout curve represent the number of bytes have been played by the client during the interval of time [13]. Thus, the playout interruption affects the smoothness of video playback. In general, the playout curve clarifies the performance of an AMP technique which smooth playout curve indicates a good AMP technique performance.

\subsection{Simulation Results}

This study investigates the playout interruption, variance of distortion of playout and the cumulative playout data for various AMP techniques. Figure 6 shows the frequency of playout interruptions for all playout control techniques. The frequency of playout interruptions substantially increased, especially at high cross traffic load values. However, the results of the FLAMP outweighs the other existing techniques in term of the frequency of playout interruption. It gives around $53.4 \%$ comparing to No Control technique, and $21.6 \%$ comparing to APTA technique.

Figure 7 demonstrates the variance of distortion of playout for all AMP. Since the fuzzy logic imitates human brain, the playout rate adaptation is perfectly tuned. Therefore, The FLAMP technique manipulates the playout rate efficiently based on network quality. The FLAMP result gives $26.5 \%$ less distortion of playout comparing to APTA and $46 \%$ comparing to No Control. 
Figure 8 illustrates to the playout curves of AMP techniques. As mentioned before, the low perversion is the most accurate technique. The playout curve of FLAMP technique is the lowest as compared to existing techniques. Consequently, this result indicates the reliability of previous results, the interruption of the video playout result and the visual quality degradation result.

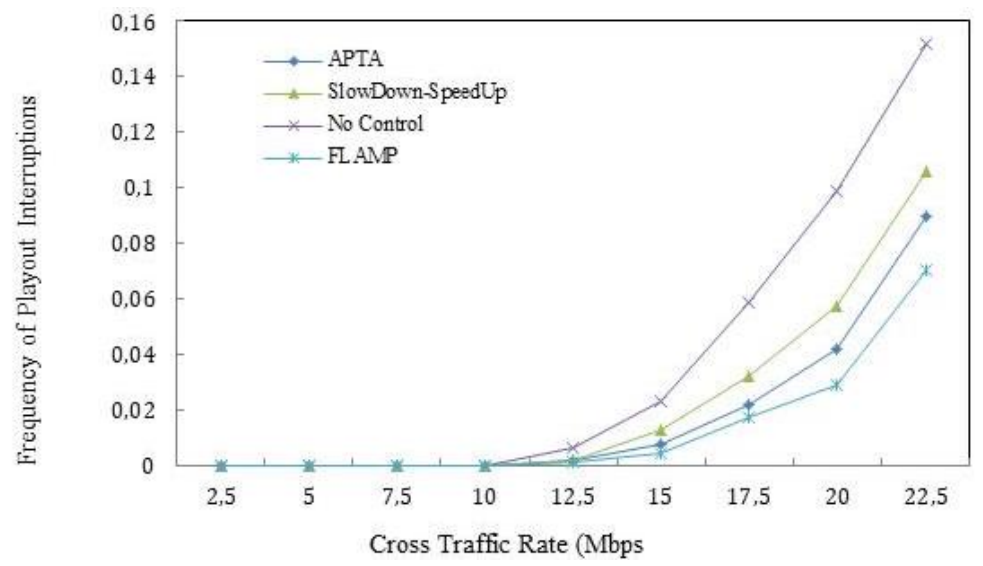

Figure 6. The frequency of playout interruption for AMP techniques

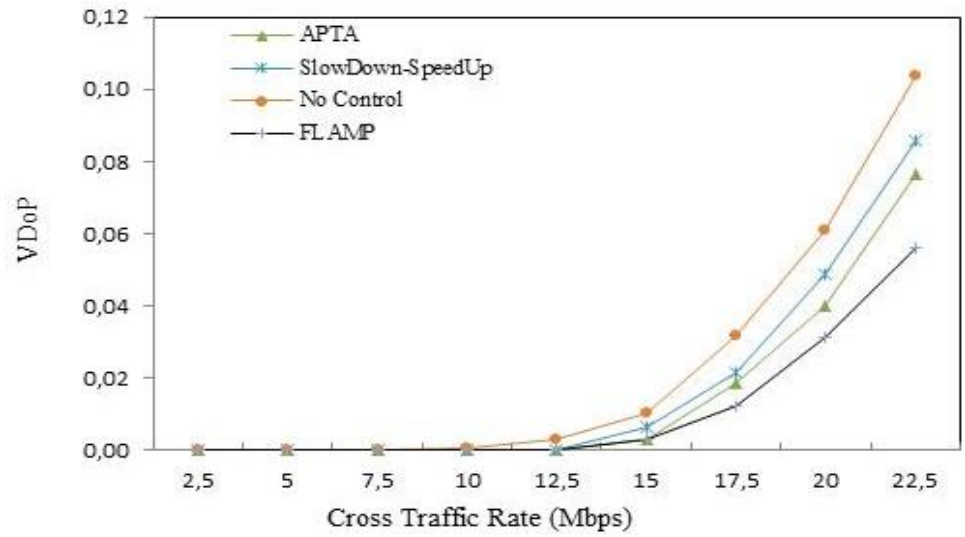

Figure 7. The variation of distortion of playout for AMP techniques

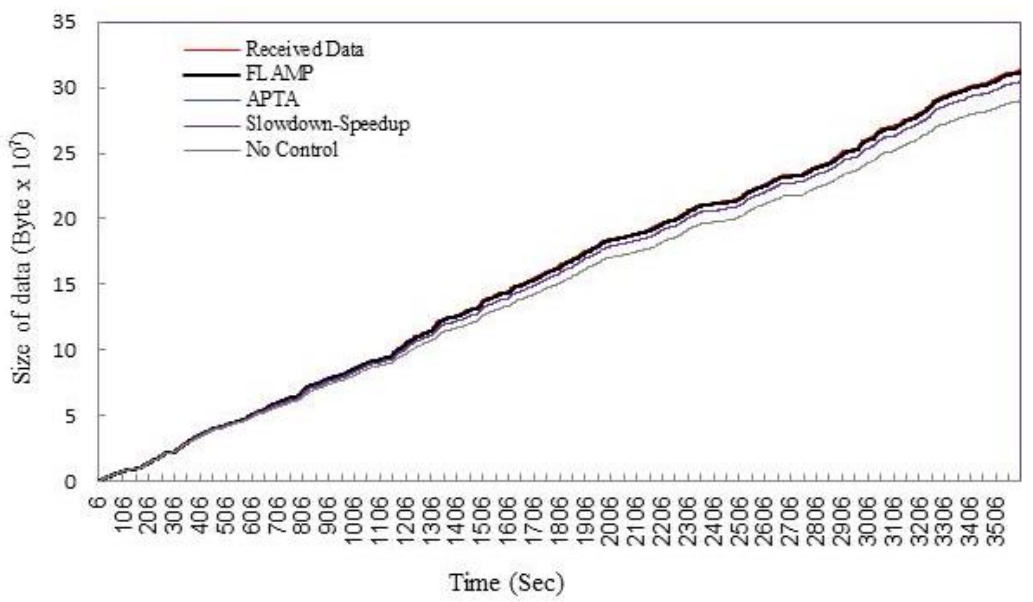

Figure 8. The playout curves for AMP techniques 


\section{CONCLUSION}

In this study, a new AMP technique based on the FL control is proposed and evaluated. The technique, FLAMP, estimates the playout rate based on the network quality. Moreover, the FL concept is fused with the AMP technique to trigger and interpolate playout rate. Numerical results indicate that FLAMP technique gives better performance in term of reducing playout interruption, visual quality degradation and smoothness of playout.

\section{REFERENCES}

[1] Al-Zoubi, H., et al., Asurvey On Recent Advances in IPTV. JJCIT, 2, (2): p. 86-106. (2016).

[2] Wu, J., et al., Content-aware concurrent multipath transfer for high-definition video streaming over heterogeneous wireless networks. TPDS, 27, (3): p. 710-723. (2016).

[3] Su, G.-M., et al., QoE in video streaming over wireless networks: perspectives and research challenges. WN, 22, (5): p. 1571-1593. (2016).

[4] Su, G.-M., et al., QoE in video streaming over wireless networks: perspectives and research challenges. QoE in video streaming over wireless networks: perspectives and research challenges: p. 1-23. (2015).

[5] Zhang, X. and H. Hassanein, A survey of peer-to-peer live video streaming schemes - An algorithmic perspective. Computer Networks, 56, (15): p. 3548-3579. (2012).

[6] Lindeberg, M., et al., Challenges and techniques for video streaming over mobile ad hoc networks. Multimedia Systems, 17, (1): p. 51-82. (2011).

[7] Hoßfeld, T., et al. Quantification of YouTube QoE via crowdsourcing. in 2011 IEEE International Symposium on Multimedia (ISM), . IEEE. (2011).

[8] Kalman, M., E. Steinbach, and B. Girod, Adaptive media playout for low-delay video streaming over error-prone channels. TCSVT, 14, (6): p. 841-851. (2004).

[9] Chen, Y. and G. Liu. Adaptive media playout assisted rate adaptation scheme for HTTP adaptive streaming over lte system. in Multimedia \& Expo Workshops (ICMEW), 2016 IEEE International Conference on. IEEE. (2016).

[10] Yang, J., et al., Online buffer fullness estimation aided adaptive media playout for video streaming. IEEE Transactions on Multimedia, 13, (5): p. 1141-1153. (2011).

[11] $\mathrm{Hu}, \mathrm{H}$., et al., Scene aware smooth playout control for portable media players over random VBR channels. T-CE, 56, (4): p. 2330-2338. (2010).

[12] Su, Y.-F., et al., Smooth control of adaptive media playout for video streaming. IEEE Transactions on Multimedia, 11, (7): p. 1331-1339. (2009).

[13] Li, M., T.-W. Lin, and S.-H. Cheng, Arrival process-controlled adaptive media playout with multiple thresholds for video streaming. Multimedia Systems, 18, (5): p. 391-407. (2012).

[14] The Network Simulator - $n s$-2. Available from: http://www.isi.edu/nsnam/ns/.

[15] MPEG-4 and H.263 Video Traces for Network Performance Evaluation. Available from: http://www2.tkn.tuberlin.de/research/trace/trace.html. 